\title{
Taking the PubChem web sketcher to the next level
}

\author{
Wolf D Ihlenfeldt \\ From 8th German Conference on Chemoinformatics: 26 CIC-Workshop \\ Goslar, Germany. 11-13 November 2012
}

The input of chemical structures in the context of Web applications is a well-known interface problem in chemical information processing. Currently, this is typically achieved by using either Java applets, or platform- and browser-specific plug-ins - both approaches require installation of custom software components which may not be possible in many use cases. Pure client-side JavaScript solutions have recently been presented, but these require the latest generation of browsers, and are significantly limited in the functionality they can provide.

The first popular Web-bases structure sketcher to break out of these moulds was the PubChem structure input tool. It is based on a server-based image streaming model, backward-compatible down to IE6 in a fully browser-and platform-agnostic fashion, and usable without any installation of client software. This software has been in continuous development since it was first deployed about five years ago. The latest version, which still maintains exceptional legacy browser compatibility, has evolved into an information hub which can directly query dozens of Internet chemistry databases. It now supports advanced HTML5 features, such as drag\&drop of structure data files and pasting of ChemDraw or ISIS/Draw data on the clipboard. An innovative operation mode for use on touchscreens with limited resolution and no mouse, and voice control for structure input on small devices or for handicapped users are other recently added features.

Published: 22 March 2013

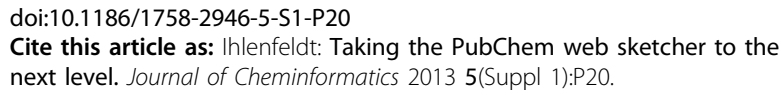

Correspondence: wdi@xemistry.com

Xemistry GmbH, Königstein, 61462, Germany

\section{Publish with ChemistryCentral and every scientist can read your work free of charge \\ "Open access provides opportunities to our colleagues in other parts of the globe, by allowing anyone to view the content free of charge." \\ W. Jeffery Hurst, The Hershey Company. \\ - available free of charge to the entire scientific community \\ - peer reviewed and published immediately upon acceptance \\ - cited in PubMed and archived on PubMed Central \\ - yours - you keep the copyright \\ Submit your manuscript here: \\ http://www.chemistrycentral.com/manuscript/

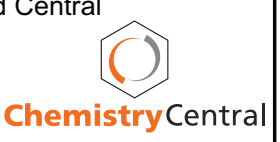

